\title{
Tangence
}

\section{Bible et littérature}

\section{Gérard Rochais}

Numéro 35, mars 1992

Des écritures à lire

URI : https://id.erudit.org/iderudit/025701ar

DOI : https://doi.org/10.7202/025701ar

Aller au sommaire du numéro

Éditeur(s)

Tangence

ISSN

0226-9554 (imprimé)

1710-0305 (numérique)

Découvrir la revue

Citer cet article

Rochais, G. (1992). Bible et littérature. Tangence, (35), 87-103.

https://doi.org/10.7202/025701ar d'utilisation que vous pouvez consulter en ligne.

https://apropos.erudit.org/fr/usagers/politique-dutilisation/ 


\section{Bible et littérature}

\section{Gérard Rochais}

L'Ancien et le Nouveau Testament sont le Grand Code de l'Art

William Blake

La question de la Bible - de sa lecture, de sa connaissance, de son utilité - interroge bien des modernes. Et non seulement les exégètes, les clercs et les étudiants en théologie ou sciences religieuses, pour qui l'étude du Livre s'impose comme base de toute culture religieuse ou théologique, mais les gens cultivés qui, naguère encore peu sensibles aux données bibliques, s'aperçoivent avec effarement que quelques-unes des grandes références de la culture, en tête desquelles se trouve la Bible, sont en train de s'effacer de la mémoire des jeunes générations, peut-être tout simplement parce que ces références n'y ont jamais pénétré.

C'est du Canada anglais que la question même de l'utilité de la Bible pour l'étude de la littérature est partie. En 1981, le grand critique litteraire torontois, Northrop Frye, publia The Great Code: Bible and Literature. Il s'était aperçu, en effet, dans ses cours et conférences, que ses étudiants, voire même ses collègues les plus jeunes, ne connaissaient plus le contenu de la Bible et ne savaient plus lire cet embarrassant volume. Les références à l'histoire de Joseph, à la sortie de l'Égypte, à la théophanie du Sinaï, aux paraboles des ouvriers de la onzième heure, du fils prodigue, n'allumaient aucune étincelle dans l'intelligence des auditeurs. Cette ignorance de la Bible par. des esprits intelligents qui voulaient acquérir une éducation supérieure dans le domaine des humanités posait problème aux universités très britanniques du Canada anglais: comment en effet rendre intelligible la littérature de langue anglaise, si on ignore le B-A-BA de la Bible? Shakespeare, Chaucer, Blake, Milton, James, Joyce ne peuvent être entièrement compris et appréciés par des lecteurs non familiers de la Bible. C'est ainsi par exemple que Les ailes de la colombe de Henry James emprunte non seulement son titre, mais également sa charge émotionnelle au psaume 55: 
88

Si encore un ennemi m'insultait,

je pourrais le supporter.

[...]

Mais toi, un homme de mon rang

mon ami, mon intime.

[...]

Plus onctueuse que la crème est sa bouche.

[...]

Ses discours sont plus doux que l'huile.

La même question de la Bible et de la littérature allait rebondir en France l'année suivante. En septembre 1982, la célèbre revue Esprit publiait un numéro intitulé: La Bible dans tous ses états. D'entrée de jeu, les responsables du numéro déclarent :

Notre question est [...] de voir comment le Livre travaille un tissu culturel, comment le Livre nous travaille, comment nous travaillons en collaboration avec lui: ici nous ne pouvions que nous trouver en champ de littérature. Entre les Saintes Lettres et les Lettres tout court, les nôtres, il y a un rapport si intime et si méconnu que nous nous sommes essentiellement attachés à le cerner. Nous n'avons pas fini de nous demander en fonction de quelles causes - l'approche scientifique et critique étant ici sans doute davantage signe que cause - une esthétique nous faisait si cruellement défaut pour lire la Bible. (p. 1)

Quelques années plus tard, un frémissement saisissait le monde très laïque de l'éducation en France; avec inquiétude, on s'avisait en effet, que tout un pan de la culture - la Bible - qui, prise dans sa totalité, avait servi de matrice à l'imaginaire littéraire et iconographique occidental, s'était totalement évanoui de la mémoire des jeunes générations ${ }^{1}$. Dans les instructions officielles destinées aux collèges et aux lycées, le ministère de l'Éducation donnait des consignes pédagogiques très précises pour que dans ces écoles foncièrement laïques on fit une lecture culturelle de la Bible. Il lui semblait important de restituer parmi la multiplicité des savoirs contemporains que doit acquérir l'étudiant une pièce manquante dont l'effacement avait fini par rendre problématique la pratique d'autres disciplines telles que la littérature, l'histoire ou l'histoire de l'art.

Le Québec serait-il épargné par cette remise en question? On pourrait le penser a priori, puisque, à la différence des écoles françaises, les écoles primaires et secondaires offrent des cours de caté-

1 Cf. Anne-Marie Pelletier, • La Bible comme mémoire culturelle *, Etudes, no 3743 (mars 1991), p. 381. 
chèse, et que quelques cégeps - tous demain? - dispensent des cours de religion. Mais quelques remarques s'imposent: les cours d'enseignement moral et religieux catholique sont très peu fondés sur la Bible. D'autre part, ces cours sont beaucoup plus tournés vers l'enseignement religieux proprement dit $>$ l'éducation chrétienne, que vers le culturel: les liens entre religion et culture sont a peu près absents des manuels officiels de catéchèse. Comment l'étudiant alors pourra-t-il faire ce lien de lui-même? La culture biblique de l'étudiant qui arrive à l'université dans une faculté de théologie ou un département de sciences religieuses est à peu près nulle, comme j'ai pu le constater dans mon enseignement de la Bible au Département des sciences religieuses de l'UQAM.

D'autre part, si l'on considère l'insatisfaction des étudiants universitaires à l'égard des cours de catéchèse reçus plus tôt; si l'on tient compte aussi de la montée du pluralisme, de la variété des religions, anciennes et nouvelles, surtout dans les grandes villes, on en vient à se demander si à ce vide dans la culture religieuse on ne devrait pas remédier par un enseignement de l'ahistoire des religions a à l'école, laissant aux différentes religions le soin de former leurs propres adeptes. Au Québec, il est vrai, les parents préfèrent s'en remettre à l'école plutôt qu'à l'Église ou aux familles, pour transmettre ce qu'ils considèrent comme un héritage familial, comme vient de le montrer Micheline Milot dans sa thèse et son livre: Une religion à transmettre. Le choix des parents (1991). ll n'y a pas de réponses toutes faites au problème de l'enseignement religieux dans les écoles au Québec; avec un peu d'imagination, on pourrait penser que cet enseignement pourrait varier selon les régions, et selon les écoles. Quoi qu'il en soit, la question du rapport de la Bible à la culture et à la littérature québécoise mérite d'être posée, car il est impossible de comprendre des films comme le Jésus de Montréal de Denys Arcand, ou les romans de Victor Lévy-Beaulieu, d'Anne Hébert, sans un minimum de culture biblique ${ }^{2}$.

Je voudrais dans cet article aborder trois questions qui s'enchaînent l'une à l'autre. La première: que signifie, dans une société puissamment et diversement sécularisée, cette demande culturelle qui se manifeste à l'égard de la Bible? Que veut dire se réapproprier la Bible au titre d'une référence culturelle, comme

2 André Sirois, - Anne Hébert et la Bible -, Voix $\varepsilon$ images, 13 (printemps 1988), p. $459-472$. 
réservoir pour l'imaginaire, matrice mythologique des productions de l'esprit occidental? Quel est le statut du texte biblique dans notre société technique ou postmoderne? Quelles relations la société entretient-elle encore avec la croyance surannée en "l'histoire sainte.? Deuxième question: pourquoi ou en quoi la Bible est-elle, selon l'expression de William Blake, le grand code de l'art, et surtout de la littérature? Qu'y a-t-il dans la Bible qui attire particulièrement les poètes et les autres créateurs artistiques du monde occidental? Ou tout simplement, quelle est la place de la Bible dans la vie humaine? Dernière question: comment peuton lire la Bible culturellement? Cette lecture suffit-elle?

\section{Que signifie cette réappropriation de la Bible comme donnée culturelle?}

Vouloir, dans une société occidentale fortement sécularisée, revenir à la Bible comme référence culturelle, dit nécessairement quelque chose de la relation que la société a avec sa propre culture et avec la croyance. La Bible, en Occident, est autre chose qu'un fragment de culture; comme peuvent l'être à nos yeux les grands mythes sumériens ou babyloniens, la Bhagavad-gita ou les écrits confucéens. La Bible, qu'on lisait alors en latin, a forgé toute la culture occidentale jusqu'à la Réforme, quoiqu'il y eût aussi au Moyen Âge - et cela on l'ignore - des traductions françaises de la Bible, très belles, fortes, juteuses, comme le montre la traduction du premier verset du Prologue de saint Jean:

Au commencement estoit Parole

Et Parole estoit envers Dieu.

Juste avant la Réforme, naquit l'imprimerie qui permit la lecture individuelle. En Angleterre, en Allemagne, les effets de la Réforme individualiste et la naissance d'une civilisation écrite s'épaulent: on traduit la Bible en langue vernaculaire, dans une langue splendide, qui va devenir la matrice de la littérature des pays anglo-saxons; on attend la même chose des pays latins, et Érasme d'écrire: "Si l'on m'en croit, l'Évangile sera lu des laboureurs, des forgerons, des maçons, des tisserands, même des filles publiques et de leurs ruffians, même des Turcs enfin.. Espoir déçu, hélas! le Concile de Trente allait interdire la traduction de la Bible en langue vernaculaire et imposer aux pays latins restés sous l'obédience catholique (France, Italie, Espagne), au lieu du pain fort et du vin savoureux de la Bible, un catéchisme étriqué. 
La Bible était réservée aux clercs et, au peuple, la doctrine efflanquée des catéchismes! Il y eut dans le monde francophone une exception: la traduction en français de la Bible par le janséniste Louis-Isaac Lemaître de Sacy, lui-même neveu de la *Mère Angélique ", la grande réformatrice de l'Abbaye de Port-Royal. Malgré la prison (1666 - 1668) et malgré la mort (en 1684), Lemaître de Sacy avait lancé l'une des plus grandes entreprises littéraires du dix-septième siècle. Une Bible comparable à celle de Luther en Allemagne, à celle du roi Jacques en Angleterre. Cette Bible qui occupa nombre de tables de chevet deux siècles durant, de Louis XIV à la Troisième République en France, fut celle de Victor Hugo, de Stendhal, de Rimbaud. La plus littéraire, èlle vient d'être rééditée par Robert Laffont, dans la collection - Bouquins. 11 fallut attendre la seconde moitié du vingtième siècle, pour que de bonnes traductions de la Bible en français voient le jour: Bible de Jérusalem et Traduction œcuménique de la Bible pour les chrétiens; la Bible très philologique de Dhorme pour les lettrés; la Bible du rabbinat ou de Chouraqui pour les juifs. Entre temps, Racine, Pascal, Proust lisaient leur Bible dans la Vulgate latine... ainsi que la majorité des séminaristes jusqu'à la fin du Concile Vatican II!

Dans ce contexte que signifie la mise en marché, toujours florissante, de la Bible? Que le peuple chrétien, catholique surtout, clercs et laïques, s'est réapproprié le livre qui est à la base même de sa religion. Même si l'on ressent parfois un certain essoufflement, on ne peut passer sous silence qu'il existe encore au Québec de nombreux cercles bibliques qui, par des approches bien variées, selon des méthodes bien différentes, tentent de pénétrer la vérité du Livre dont saint Augustin vantait la douceur, ces "chastes délices de l'âme", et d'y découvrir la Parole fondatrice de leur foi. Plus de chrétiens que jadis utilisent la Bible, en lisent des fragments pour leur méditation ou leurs réflexions personnelles. Enfin, il n'est pas si difficile de savoir qu'au-delà des frontières du monde occidental, surtout parmi les opprimés, en Amérique latine, on scrute la Bible pour y trouver la force de vivre, jusqu'au péril de la prison, de la torture et de la mort. On endure la prison, parce qu'on la lit et que cette lecture est jugée subversive par des autorités politiques qui s'affichent pourtant comme chrétiennes. On est assassiné même, comme $\mathbf{M}^{\mathrm{gr}}$. Oscar Romero, parce qu'on prêche et témoigne de la vérité révélée aux hommes par ce livre. L'interprétation engagée du texte biblique fait alors lever son sens, prête son souffle au texte. 
Moins qu'aucun autre texte la Bible ne dispense de l'engagement et donc du risque de l'interprétation. Le texte ne tranche, en effet, que pour celui qui prend le risque d'interpréter. La théorie littéraire moderne rappelle que l'identité d'un texte consiste en ce qu'il dit, mais également dans la manière dont il est lu, dans l'histoire des lectures et des usages qu'il autorise. Il n'est pas nécessaire de fréquenter les Églises pour savoir que des communautés lisẹnt ce texte comme principe de leur rassemblement et comme norme de leur agir. La Bible est d'abord un texte confessionnel, que diverses communautés chrétiennes lisent en l'intégrant à une action liturgique qui déclare que cette mémoire culturelle est une présence sacramentelle. Mais la Bible est aussi, dans notre société sécularisée, une référence culturelle. Qu'est-ce que lire la Bible comme référence culturelle? Que veut-on y lire? Comment lira-t-on? Ce regard culturel porté aujourd'hui sur la Bible, sa redécouverte à travers les ouvres qu'elle a inspirées ou suscitées au long des siècles, pas plus que sa confrontation avec les questions actuelles qui se posent à notre société, ne peut être considéré comme vain ou inutile. * La Bible, écrit Paul Beauchamp, est faite pour être déchiffrée et résonner au milieu des autres lettres et de leur fait: il n'y a pas à craindre qu'elle y perde sa tonalité propre. ${ }^{3}$ La référence strictement culturelle à la Bible signifie, a mon avis, au moins deux choses apparemment contradictoires: elle dit d'abord mémoire de notre culture, et aussi oubli de ce qui fonde cette culture: l'alliance de Dieu avec l'humanité et le cosmos, révélée dans la religion judéo-chrétienne.

La Bible est au cœur de la culture occidentale, au cœur de la culture québécoise, comme l'a rappelé récemment une exposition montée conjointement par le Musée de la Civilisation à Québec et SOCABI (Société catholique de la Bible) qui s'est tenue du 27 mars au 27 août 1991. Elle était intitulée: La Bible entre mots et images. L'exposition permit au visiteur de découvrir les diverses présences bibliques, par l'écrit ou l'image, dans la culture occidentale. Elle comprenait trois volets: dans le premier, l'exposition présentait l'importance de la Bible dans la tradition juive. Le deuxième volet permettait de découvrir la présence de la Bible au Moyen Âge, la diversité des éditions et des traductions de ce livre et enfin la place importante qu'on lui réservait dans la tradition protestante. Le troisième et dernier volet de l'exposition

3 Paul Beauchamp, L'un et l'autre Testament, t. 2, Paris, Seuil, 1990, p. 91. 
montrait la diversité des moyens utilisés au cours de l'histoire pour représenter les grands thèmes bibliques, avec une préoccupation plus insistante pour les présences bibliques dans le contexte culturel québécois. Car l'influence de la Bible sur la culture québécoise traditionnelle et contemporaine est plus considérable que l'on se l'imagine habituellement.

La culture québécoise, teintée aujourd'hui de multiculturalisme, a puisé ses inspirations dans le contenu biblique transmis dans l'art, l'imagerie populaire, le langage, les productions culturelles comme la littérature, les téléromans, la publicité ou le cinéma. Cette exposition permit donc aux visiteurs de découvrir que la Bible faisait aussi partie du patrimoine culturel québécois, de sa tradition à laquelle chacun, qu'il le veuille ou non, est exposé. . On continue toujours à croire, écrit Heidegger, que la tradition est passée et qu'elle n'est plus qu'un objet de la conscience historique. On continue toujours à croire qu'elle est ce que nous avons proprement derrière nous, quand elle vient au contraire vers nous parce que nous sommes exposés à elle et qu'elle est notre destin. ${ }^{4}$

Si la culture est, selon le mot d'Édouard Herriot, ce qui reste quant on a tout oublié, il faut reconnaître que l'intérêt culturel pour la Bible traduit une perte, un oubli. L'oubli que la Bible est aussi une "Parole " et, pour le croyant, "Parole de Dieu * qui dévoile et réalise aussi ce qu'elle annonce. Le peuple d'Israël, et les chrétiens à sa suite, ont la conviction que Dieu s'est dévoilé dans l'histoire concrète, quotidienne, charnelle, sociale et politique d'Israël, puis pour les chrétiens dans la vie et le destin de JésusChrist, dans le témoignage qu'en ont rendu les premières générations chrétiennes, et finalement dans l'interprétation sans cesse renouvelée de ce témoignage. La Bible n'est pas forcément un livre religieux ou édifiant. Il ne faut pas trop se surprendre que pendant des siècles la censure ecclésiastique ait voulu "protéger" les chrétiens de sa lecture. Pourtant, au sein de ses paroles les plus banales, la Bible transmet et décode une communication, celle d'une alliance avec Dieu, commencée avec le peuple d'Israël et pleinement réalisée en Jésus-Christ. Elle dévoile un Dieu qui parle et qui s'est engagé dans une histoire de libération. Ce dévoilement ne va pas de soi, ni ne s'impose. Il suppose un 
regard de foi, une interprétation. Culturellement, il demeure une énigme. La Bible reste une question ouverte. L'intérêt purement culturel qu'on lui porte peut signifier aussi l'oubli du sens qu'au cours des siècles, et aujourd'hui encore, les croyants, juifs ou chrétiens, lui ont prêté et continuent de lui donner.

\section{Le Grand Code}

- L'Ancien et le Nouveau Testament. sont le Grand Code de l'Art ", a écrit William Blake dans les annotations qui entourent sa gravure du Laocoon. Northrop Frye a repris cette expression comme titre de son livre sur la Bible et la littérature après avoir, dit-il, médité de nombreuses années sur ce qu'elle voulait dire. Dans son livre, *il tente d'étudier la Bible du point de vue d'un critique littéraire. Il veut introduire le lecteur moyen à une connaissance de la Bible et à certaines manières d'appliquer cette connaissance au reste de ses lectures*. Le premier chapitre traite du langage qu'emploient les gens pour parler de la Bible et de questions qui s'y rattachent, telle que l'existence de Dieu. Ce chapitre d'ouverture "définit un contexte pour parler de la Bible en tant qu'influence sur l'imagination. Les deux chapitres suivants traitent du mythe et de la métaphore. L'auteur y définit ces termes et entend montrer que mythe et métaphore répondent à la question: *Quel est le sens littéral de la Bible?* Le chapitre sur la typologie conclut la première partie en ajoutant une dimension temporelle au raisonnement, en se rapprochant de la manière traditionnelle dont le christianisme a toujours lu sa Bible. La seconde partie traite d'une application plus directe des principes critiques à la structure de la Bible, mais dans l'ordre inverse. L'auteur part des sept phases traditionnelles de la révélation: création, exode, loi, sagesse, prophétie, évangiles et apocalypse. Il postule deux formes de vision apocalyptique, ce qui fait huit phases en tout, la huitième ramenant à la thèse centrale du rôle du lecteur. Il passe ensuite en revue, de façon inductive, d'abord l'imagerie, puis les structures narratives de la Bible. Le chapitre final aborde à nouveau la question du langage et présente une brève esquisse d'une conception polysémique du sens, appliquée à la Bible. Il tente de suggérer quelques réponses à des questions sur la direction dans laquelle on va en partant du sens *littéral *

Le Grand Code obéit, comme la Bible, à la symétrie de l'image en miroir; Langage I - Mythe I - Métaphore I - 
Typologie I - Typologie II - Métaphore II - Mythe II Langage II. Cette disposition pourtant ne pèse guère sur la progression d'une étude qui est très difficile à résumer. Il y a pourtant une unité dans le travail même de Frye. Son premier livre sur Blake (1947), Effrayante symétrie, annonce déjà le programme du Grand Code, et il n'est sans doute pas inutile d'en citer un passage pour comprendre ce que ce grand théoricien de la critique littéraire entend faire dans son dernier livre.

Le fondement de la Bible, comme celui de la saga épique, religieuse et historique, est en relation avec les dieux anthropomorphiques et les hommes théomorphiques, en partie histoire légendaire et en partie vision prophétique. Mais la Bible n'est ni une cuvre d'art unique comme l'lliade, ni une œuvre qui vaut par son extension comme la Mababharata: elle est le produit historique d'une tradition visionnaire. Elle consigne un perpétuel remaniement des visions antérieures et primitives et, au fur et $a$ mesure, elle se fait de plus en plus explicitement prophétique jusqu'à ce que les légendes compliquées d'un peuple obscur prennent la forme de la vision complète et cyclique de la chute, de la rédemption et de l'apocalypse. L'Ancien Testament débute [historiquement] par le récit de la fuite hors d'Égypte jusqu'à Canaan, sous la conduite de Moïse et de Josué, et s'achève par la recréation prophétique et allégorique de cet événement: la fuite de l'imagination hors d'une *fournaise de fer * jusqu'à la Cité de Dieu, grâce au pouvoir d'un homme divin ou d'un Messie.

Les Évangiles consolident cette vision du Messie dans la vision de Jésus, qui a le même nom que Josué, et la preuve des événements de la vie de Jésus, tels qu'ils sont relatés dans les Évangiles, est fournie non par les témoignages contemporains, mais par ce que les prophètes avaient dit de ce que serait le Messie. La reprise imaginative des visions de l'Ancien Testament dans le Nouveau, atteignant son point culminant dans la mosaïque d'allusions et de citations de l'Apocalypse, achève simplement un processus qui se déploie avec une ampleur considérable à l'intérieur même de l'Ancien Testament. ${ }^{5}$

On le voit d'après cette citation, c'est la recherche des types, comme aussi de leurs antitypes, c'est tout ce mouvement d'allées et venues d'une lecture typologique de la Bible qui justifie, en premier lieu, d'appeler ce dernier livre Le Grand Code. Il y a aussi une seconde raison: la Bible, comme suggère Frye, est aussi un code pour déchiffrer la littérature séculière, qu'elle a pénétrée par 
son texte et son esprit. La Bible en effet propose un modèle de l'espace, du ciel à l'enfer, et du temps, depuis la Genèse jusqu'à l'Apocalypse; tous les poètes occidentaux s'en sont servi, qu'ils l'aient su ou non. Mais Frye n'est pas trop loquace à ce sujet. Il pensait, à l'origine, pouvoir passer en revue d'une façon assez complète l'imagerie et la narration bibliques, puis expliquer comment ces éléments de la Bible avaient constitué un cadre de l'imaginaire, ou de l'univers mythologique, à l'intérieur duquel la littérature occidentale avait fonctionné jusqu'à aujourd'hui encore, dans une large mesure. Il n'a pu donner suite à ce projet, ce premier volume ayant pris trop d'ampleur. Il annonce néanmoins un second volume qui traiterait de ces questions. Je ne sais s'il a pu y parvenir avant sa mort.

Néanmoins, déjà dans ce premier volume, Frye répertorie les métaphores les plus persistantes de la tradition occidentale et les renvoie à la Bible: il enregistre et classe les intrigues favorites de deux mille ans d'histoire occidentale. Il a des intuitions êtincelantes du genre de celle-ci: Le procès de Kafka "est à lire comme une espece de midrash sur le livre de Job. Car toute textualité pour lui est intertextualité. Et *la vraie différence entre le poète original et le poète imitateur est simplement que le premier est plus profondément imitateur. - Le nouveau poème, comme l'enfant nouveau-né, prend place dans un ordre verbal déjà existant $[. .$.$] on ne peut faire de la poésie qu'à partir d'autres$ poèmes; des romans qu'à partir d'autres romans.. En traitant de Saül qui a épargné son ennemi le roi Agag pour s'entendre dire qu'en ne le tuant pas il frustre son dieu féroce d'un sacrifice et que cela ne lui sera pas pardonné par une divinité qui "n'est pas un homme pour se repentir * (I S 15,29), Frye écrit:

Il me semble que le narrateur [...] par une sorte de gaffe inspirée, s'est arrangé pour ajouter l'élément qui rend authentiquement tragique l'histoire de Saül: l'idée d'une méchanceté à l'intérieur même de la nature divine, idée qui est peut-être essentielle a toute grande tragédie. Saül devient a partir de ce moment un homme condamné qui a des accès intermittents de mélancolie et d'efforts frénétiques mais futiles pour se débarrasser de la menace que représente David. Dans la scène de la magicienne d'Endor, il se tourne en fin de compte vers les puissances occultes dont il a lui-même interdit la consultation, et désastre et mort s'ensuivent. Cette déchéance terrible et inévitable rappellera au lecteur moderne que Shakespeare a certainement étudié avec beaucoup de soin le técit de la vie de Saül avant d'écrire Macbeth. 
Quelques autres allusions à Milton, Byron, Blake illustrent le texte, mais manifestement, Frye n'a pas prouvé que la Bible est. le Grand code qui a constitué l'univers mythologique des artistes occidentaux. Le second volume, promis par l'auteur, aurait sans doute comblé ce manque.

La Bible est le Grand Code de l'art surtout parce qu'elle permet une lecture typologique. Le théoricien de la critique littéraire peut faire, certes, de la Bible l'objet de son commentaire puisque celle-ci ùtilise un grand nombre de modes littéraires et qu'elle présente à profusion la métaphore, la métonymie, le symbole, l'analogie et bien d'autres figures de langage pour rendre compte de l'histoire humaine, de la réflexion sur soi: création, exode, sagesse, loi, prophétie, évangile et apocalypse. L'apport fondamental de Frye est de montrer que la Bible est le Grand Code parce qu'elle doit être lue comme un jeu de types et que c'est en fonction d'un ensemble de types repris et illustrés que les auteurs bibliques écrivaient. Les pages les plus nourrissantes me semblent bien être celles qui, au centre du volume, concernent la typologie (p. 130-200).

L'auteur commence par une affirmation qui, pour être juste en partie, manque de nuances:

Comment savons-nous que l'histoire de l'Évangile est vraie? Parce qu'elle confirme l'Ancien Testament. Mais comment savons-nous que les prophéties de l'Ancien Testament sont vraies? Parce qu'elles sont confirmées par l'histoire de l'Évangile. Le prétendu témoignage est renvoyé d'un testament à l'autre comme une balle de tennis; et aucun autre témoignage ne nous est donné. Les deux Testaments forment un miroir double, chacun réfléchissant l'autre, mais aucun des deux ne réfléchissant le monde extérieur.

C'est juste, mais avec exagération. Les auteurs du Nouveau Testament, il est vrai, sollicitent souvent le texte de l'Ancien Testament pour l'adapter à la vie de Jésus, mais souvent aussi ils interprètent la vie de Jésus pour la faire concorder aux prédictions de l'Ancien Testament, sans que jamais on ait une adéquation parfaite. Citant saint Augustin, Frye écrit: -Le Nouveau Testament est caché dans l'Ancien Testament; l'Ancien est dévoilé dans le Nouveau *; c'est vrai, mais il ne faudrait pas forcer la dose! Le meilleur exemple de ce qu'on appelle type est peut-être donné par le texte de Genèse 1,3: •Dieu dit: "Que la lumière soit!" ", qui trouve son antitype dans le Prologue du quatrième 
évangile: « ̊̀ l'origine était le Verbe... Tout fut par lui... Ce qui a été manifeste en lui, c'était la Vie. (Jn 1,1-4). Reprise du texte de la Genèse par Jean, c'est juste; mais corrigée, car la Vie chez Jean ne désigne pas la vie naturelle, mais bien la Vie spirituelle ou éternelle, dont il est question dans tout son Évangile. L'Exode constitue le recueil par excellence des types de la Bible tout entière, et Frye peut écrire que a mythiquement, l'Exode est la seule chose qui arrive véritablement dans l'Ancien Testament. Oui, mythiquement: les douze tribus ont leur antitype dans les douze apôtres choisis par Jésus pour régner sur l'Israël idéal; le passage de la mer Rouge a sa correspondance dans le baptême; la manne préfigure l'eucharistie, la Loi donnée à Moïse annonce le "Sermon sur la montagne - donné par Jésus lui-même; la Cène est sans doute un repas pascal et la Résurrection est, pour une part, la victoire sur les ennemis d'Israël.

C'est ainsi que la Bible a toujours été traitée dans la liturgie par la tradition juive et la tradition chrétienne, bien que de manière différente, car ce qui constitue parfois pour les chrétiens un antitype réalisé demeure pour les juifs croyants à accomplir, que l'on songe simplement aux prophéties messianiques. Le rapport type-antitype s'exprime complètement dans les prières et le rituel pascal du Seder, dans la liturgie chrétienne, l'Exultet du samedi saint représente peut-être la célébration la plus somptueuse de cette relation: "Ô péché véritablement nécessaire d'Adam... Ô faute bienheureuse... Ô nuit vraiment bénie... "

Selon Frye, "l'organisation typologique de la Bible présente à un critique littéraire la difficulté d'être unique en son genre: aucun autre livre au monde n'a [...] une structure qui ressemble même de loin à celle de la Bible chrétienne [...] Naturellement, comme elle est la manière tout indiquée et évidente de lire la Bible, et les érudits étant ce qu'ils sont, la typologie est une matière négligée, même en théologie, et on la néglige aussi en dehors de la théologie parce qu'on la suppose liée à un attachement doctrinaire au christianisme. La question de savoir pourquoi tant de gens omettent la tradition typologique, en dépit de son utilisation liturgique demeure controversée. La typologie que Frye place à la base de sa lecture de la Bible ne nous livre pourtant que le squelette de l'Écriture. Il y a de nombreux procédés constitutifs de la richesse et de la densité de la Bible. Frye relève la métaphore en tant que mode déterminant du discours biblique. On peut penser aussi à l'imaginaire du paradis terrestre, 
un imaginaire d'oasis, d'arbres et d'eau qui attire, remarque l'auteur, tout particulièrement ceux qui étaient originairement des nomades du désert. La Bible évolue aussi entre les images de la vie pastorale: bergers, troupeaux, bons bergers, etc - et celles de la ville: Jérusalem, la ville de David, la colline du temple, voué à la profanation et à la destruction, lieu où la colère et la justice de Dieu s'expriment par la destruction et la reconstruction, et enfin .la Jérusalem d'en-haut... qui est notre mère. (Ga 4,26). Frye ne veut pas réduire la métaphore à une comparaison condensée ou à un simple souvenir métonymique. Pour lui, c'est toute la Bible qui a une structure en miroirs, chacun reflétant l'autre, et ce que les écrivains bibliques disent se réfère dans un premier sens, même lorsque l'intention de l'écrivain semble historique, à d'autres passages de la Bible. Sa fonction est de nous élever, par-delà la foi, à la vie supérieure de la vision. Il écrit:

La Bible paralyse délibérément toute tentative de mouvement vers un référent: elle n'est pas un livre indiquant une présence historique en dehors d'elle, mais un livre qui s'identifie à cette présence même. À la fin, le lecteur lui aussi est convié à s'identifier au livre. Milton suggère que l'autorité suprême de la religion chrétienne vient de ce qu'il appelle la Parole de Dieu dans le cour, supérieure à la Bible elle-même car pour Milton, ce - cœur * n'appartient pas au lecteur subjectif, mais au Saint-Esprit. Ainsi le lecteur achève l'opération visionnaire de la Bible en se débarrassant à la fois de l'illusion subjective et de l'illusion objective. L'Apocalypse est ce à quoi ressemble le monde une fois que l'ego a disparu.

En dépit de ses inexactitudes et contresens manifestes, en dépit de ses difficultés aussi, ce livre n'en demeure pas moins magnifique, rempli d'idées piquantes, de jugements excitants, de rapprochements suggestifs. Il rappelle quelques principes fondamentaux de l'interprétation biblique, et pose des problèmes et des questions de première importance pour les critiques littéraires, les exégètes et, d'une manière générale, pour le public cultivé. Il invite surtout à une lecture - différente - de la Bible, culturelle.

\section{Comment lire la Bible comme référence culturelle?}

À la fin de son volume, Frye plaide pour le sens polysémique d'un texte, et notamment de la Bible. Il écrit: 
Lorsque nous lisons, nous avons très souvent l'impression qu'il y a encore d'autres découvertes à faire à l'intérieur de la même structure verbale. Le sentiment que nous avons est, à peu près, "qu'il y a plus de choses à en tirer., ou bien nous pouvons dire d'un texte que nous admirons particulièrement que, chaque fois que nous le lisons, il nous apporte quelque chose de nouveau. $\mathrm{Ce}$.quelque chose de nouveau n'est pas forcément quelque chose que nous n'avions pas remarqué auparavant, mais peut venir plutôt d'un nouveau contexte dans notre expérience. Cela implique que, lorsque nous commençons à lire, une certaine espèce de processus dialectique commence à se dérouler, de sorte que toute appréhension donnée. de ce que nous lisons fait partie d'une série de phases: ou de stades de compréhension.

Au Moyen Âge, ces phases étaient classées en schèmes déterminés (quatre sens scripturaires). Selon Frye, Dante donne un résumé concis et utile de la version la plus familière du schéma tout entier:

Ainsi, pour la clarté de ce que j'ai à dire, il faut savoir que le sens de cet ouvrage n'est point simple, et qu'on peut dire au contraire polysème, c'est-à-dire doué de plusieurs signifiances; car autre est le sens fourni par la lettre, et autre est le sens qu'on tire des choses signifiées par la lettre. Et le premier est dit littéral, mais le second allégorique, ou moral, ou anagogique. Cette façon de traiter les chose contées se peut considérer, pour plus de clarté, dans un verset comme celui-ci: *Quand Israël sortit de l'Égypte, et la maison de Jacob du sein du peuple barbare, la Judée fut faite sanctification du Seigneur, Israël sa puissance. * Car si nous regardons à la lettre seule, nous voyons signifiée la sortie d'Égypte des fils d'Israël, au temps de Moïse; si c'est à l'allégorie, nous voyons signifié notre rachat, par l'œuvre du Christ; si c'est au sens moral, le verset signifie la conversion de l'âme quittant le deuil et la misère du péché pour un état de grâce; si c'est au sens anagogique, il signifie la sortie de l'âme sainte hors de la servitude d'un monde corrompu, et la liberté de la gloire éternelle. Et bien que ces sens mystiques soient appelés de noms divers, tous en général peuvent être dits allégoriques, étant differents du sens littéral ou historial. ${ }^{6}$

Dante exprime, de façon claire, la théorie médiévale des quatre sens de l'Écriture. C'est un schème qui est logique et cohérent, mais il présente, selon Frye, deux caractéristiques qui 
peuvent faire hésiter si l'on songe à l'adopter comme une base de critique contemporaine, que ce soit de la Bible ou d'autre chose. Le schéma de Dante présuppose, en premier lieu, la vérité exclusive de l'une des interprétations de la Bible, celle qui l'assimile au christianisme catholique médiéval. Sur cette base, il est bien sûr impossible d'établir un dialogue suivi avec des hommes qui ont une tout autre conception du monde, et cela donne naturellement au schéma une utilité limitée pour aujourd'hui. En second lieu, note Frye, à chacun des quatre niveaux de Dante, «les mots de la structure verbale à l'étude, la Bible ellemême; sont subordonnés à quelque chose d'autre qui est supposé être plus véritable. Une fois encore, le plus véritable se trouve être quelque chose d'extérieur aux mots, qui est considéré comme leur étant supérieur*. Et pourtant Frye va défendre une lecture polysémique de la Bible, et essayer d'adapter la théorie des quatre sens à aujourd'hui. Il écrit:

Le fait de rattacher notre propre compréhension *littérale- de la Bible en tant que livre au reste de notre connaissance, plus particulièrement celle de l'arrière-plan historique et culturel de la Bible, crée une synthèse qui commence bientôt à passer au niveau de la connaissance et de la compréhension à un niveau existentiel, de la signification *allégorique - de Dante à la signification *tropologique *, d'un *ou bien * de Kierkegaard a son autre sou bien. Une telle intensification, qu'elle ait ou non quelque chose à voir avec la Bible, nous conduit de la connaissance aux principes d'action, du plaisir esthétique d'étudier un monde d'objets et de faits intéressants à ce que Kierkegaard appelle la liberté morale. Ce changement de perspective amène au mot foi.

Si la lecture polysémique de la Bible est possible, et même à recommander, la lecture culturelle de la Bible est aussi possible. Mais à quelles conditions? Dans un article fort intéressant, intitulé - La Bible comme mémoire culturelle *, Anne-Marie Pelletier énonce trois principes que je vais reprendre ${ }^{7}$. Un premier principe pourrait consister à lire - ou tenter de lire - le texte aussi intelligemment qu'il est écrit...* Il faut donc abandonner le préjugé selon lequel le texte biblique, enraciné dans les cultures anciennes, serait un texte naîf, étranger à notre mentalité scientifique et technique. Car en partant du postulat de la naïveté du

7 Anne-Marie Pelletier, - La Bible comme mémoire culturelle:, Etudes, $n^{\circ} 3743$ (mars 1991), p. 381-392. 
texte biblique, le lecteur met souvent la naïveté dans sa propre lecture et n'écoute plus le texte. On croit le connaître, lors même qu'on ne l'a jamais lu réellement et fait effort pour le comprendre. Le travail de lecture consiste à revenir au texte luimême, pour réentendre ce qu'il a à dire, sous une forme parfois déconcertante. On croit connaître, pour les avoir entendus, mais peut-être jamais lus, les premiers chapitres de la Genèse; volontiers on les jugera naiifs, sans percevoir la manière dont ces textes fondent une anthropologie, expriment la relation de Dieu à l'homme, et de l'homme au cosmos ou encore aux animaux, et situent l'efficace de la parole: "Dieu dit: Que la lumière soit, et la lumière fut. - Il faut lire simplement, en sachant néanmoins que ce modeste projet de lecture peut nécessiter qu'on y engage des forces, ne serait-ce que pour déblayer l'amoncellement de lieux communs qui encombrent l'accès au texte et sont étrangement résistants. On peut n'avoir rien lu de la Bible et *savoir. que l'Ancien Testament parle d'un Dieu vengeur et dur, alors que le Nouveau Testament présente, au contraire, un Dieu d'amour! Comme si ce n'était pas le même Dieu!

Second principe en continuité avec le précédent: non seulement lire en respectant la nature du texte, mais aussi lire en lui ce qu'il dit de lui-même, lire la mention du statut qu'il revendique. En d'autres termes, lire ce qu'il dit et lire ce qu'il déclare au sujet de ce qu'il dit - donc aussi les passages où il désigne un énonciateur divin comme source de ses énoncés. (p. 390)

Il est important de reconnaître que le texte biblique déclare si souvent un énonciateur qu'il nomme Dieu ou qu'il représente comme prophète, c'est-à-dire porte-parole de Dieu. Ces textes aussi se présentent souvent comme des adresses au lecteur, qui reste libre de répondre au texte, d'entrer dans le *nous * que lui tend le texte. Dans son article .Lire la Bible.8, Julia Kristeva remarquait que la plupart des travaux actuels sur la Bible oublient de demander: "Qui parle dans la Bible?" et "Pour qui? *. Elle commentait: "forclusion considérable - qui est celle du sujet de l'énonciation biblique et, par voie de conséquence, celle de son destinataire. La lecture ne devrait pas ignorer cette donnée, qui vaut pour bien d'autres textes que le texte bibliques.

Troisième requête enfin, liée cette fois à une donnée spécifique au texte biblique parce qu'elle concerne son mode d'écriture et

8 Julia Kristeva, * Lire la Bible *, Esprit, n 1667 (septembre 1982), p. 143-152. 
sa manière propre de gérer la temporalité. Au-delà du lieu commun qui ramène avec trop de simplicité le temps biblique à un temps linéaire, on sait que la Bible constitue en figures certains des événements qu'elle narre, de même qu'elle inscrit dans cette même dynamique tel ou tel personnage de l'Histoire vécue par Israël. (p. 390)

Le livre de Northrop Frye le montre éloquemment. La Bible est assemblée comme un tout puissamment intégré, où les différentes classes d'écrits (loi, sagesse, prophètes, apocalyptique) s'articulent les unes aux autres. Il n'existe guère de séquences, comme l'a montré Frye dans ses études sur la typologie biblique, qui n'ait le tout pour horizon. La Bible commande d'être lue en épousant ce dynamisme, en apercevant l'ouverture qui, de moment en moment, en appelle à ce qui n'est pas encore dit. Une analyse littéraire soignée, à l'aide de l'ouvrage de Frye, permet de discerner un pareil mouvement. Mais, ni la lecture exégétique moderne, ni la lecture homilétique ne portent vers ce type de réflexion. Un retour à la lecture patristique de la Bible serait très utile en ce sens.

"Lire, écrit Maurice Blanchot, exige un don qui n'est pas donné à l'avance, qu'il faut chaque fois recevoir, acquérir et perdre, dans l'oubli de soi-même. $" 9$ La lecture culturelle de la Bible exige, comme toute lecture, que l'on se soumette au texte pour entendre ce qu'il énonce derrière ce qu'on lui prête ou ce qu'on croit en savoir. Alors cette lecture rend l'écriture biblique à son tranchant, qui est aussi souvent sa plus grande proximité. Elle rend apte aussi à ressaisir les divers modes de présence de la Bible à notre tradition littéraire, et toutes les formes de son impact sur l'imaginaire occidental.

9 Maurice Blanchot, L'espace littéraire, Paris, Gallimard, coll. \& Idées *, $\mathrm{n}^{\circ} 155,1955$, p. 254. 